\title{
A NOTE ON CONVERGENCE OF WEIGHTED SUMS OF RANDOM VARIABLES
}

\author{
XIANG CHEN WANG \\ Department of Mathematics \\ Jilin University \\ Changchun \\ China \\ M. BHASKARA RAO \\ Department of Probability \& \\ Statistics, The University, \\ Sheffield S3 7RH, U.K. \\ and University of Pittsburgh \\ (Received January 3, 1984, and in revised form July 25, 1984)
}

ABSTRACT. Under uniform integrability condition, some Weak Laws of large numbers are established for weighted sums of random variables generalizing results of Rohatgi, Pruitt and Khintchine. Some Strong Laws of Large Numbers are proved for weighted sums of pairwise independent random variables generalizing results of Jamison, Orey and Pruitt and Etemadi.

KEY WORDS AND PHRASES. Independent and paimvise independent random variables, weighted sums of random variables, uniform integrability, convergence in probability, in mean, strong convergence, random elements in separable Banach spaces.

1980 AMS SUBJECT CLASSIFICATION CODE. 6OFO5, 60F15.

1. INTRODUCTION.

Let $x_{n}, n \geq 1$ be a sequence of real random variables defined on a probability space $(\Omega, \beta, P)$ and $a_{n k}, n \geq 1, k \geq 1$ a double array of real numbers. Limit theorems have been studied in the literature for the sequence $\sum a_{n k} x_{k}, n \geq 1$ of weighted sums of the sequence $x_{n}, n \geq ' 1$ under some conditions on the double array of numbers and on the distribution of the sequence $x_{n}, n \geq 1$. Jamison, Orey and Pruitt [4] studied almost sure convergence of weighted sums under the assumption that the sequence $x_{n}, n \geq 1$ is independently identically distributed with $E\left|X_{1}\right|<\infty$. One of the objects of this paper is to extend the result of Jamison, Orey and Pruitt [4] on almost sure convergence to cover the case of pairwise independent identically distrubuted sequences $x_{n}, n \geq 1$ with $E\left|X_{1}\right|<\infty$. Recently, Etemadi [3] has shown that Strong Law of Large Numbers is valid for sequences $x_{n}, n \geq 1$ which are pairwise independent identically distributed with $E\left|X_{1}\right|<\infty$. The main result of Section 3 covers Etemadi's result.

Our second objective in this paper is to study convergence in probability of the sequence of weighted sums described above. Convergence in probability has been studied under the following condition.

(B) There is a random variable $X$ on $\Omega$ such that $E|X|^{s}<\infty$ for some $s \geq 0$ and $P\left\{\left|x_{n}\right| \geq x\right\} \leq P\{|x| \geq x\}$ 
for every $x \geq 0$ and $n \geq 1$. See Rohatgi [7]. Wei and Taylor [9, Lemma 3, p. 284] have shown that if

(A) $\operatorname{Sup}_{n>1} E\left|x_{n}\right|^{r}<\infty$ for some $r>0$

holds, then ${ }^{-}(B)$ holds for every $0 \leq s<r$. In this paper, we study convergence in probability for sequences of weighted sums under the condition that

(C) $x_{n}, n \geq 1$ is uniformly integrable.

One can show that if (B) holds, then $\left|x_{n}\right|^{s}, n \geq 1$ is uniformly integrable. See Chung [2, Exercise 7, 4.5]. One can also give examples of sequences $X_{n}, n \geq 1$ satisfying (C) but not (B) with $s=1$.

2. CONVERGENCE IN PROBABILITY.

In this section, we present some results on convergence of weighted sums from which Weak Law of Large Numbers is derivable. Theorem 1 generalizes some results in the literature in this area. See the remarks following Theorem 1.

THEOREM 1 . Let $x_{n}, n \geq 1$ be a sequence of pairwise independent random variables such that $x_{n}, n \geq 1$ is uniformly integrable. Let $a_{n k}, n \geq 1, k \geq 1$ be a double array of real numbers salisfying

(i) $\sum_{k \geq 1}\left|a_{n k}\right| \leq C$ for every $n \geq 1$ for some constant $C>0$ and

(ii) $\max \left|a_{n k}\right|, n \geq 1$ converges to 0 . $\mathrm{k} \geq 1$

Then $\sum_{k \geq 1} a_{n k}\left(X_{k}-E X_{k}\right), n \geq 1$ converges to 0 in the mean. $\mathrm{k}>1$

PROOF. It is clear that the series $\sum a_{n k}\left(X_{k}-E X_{k}\right)$ converges absolutely a.e. $\mathrm{k} \geq 1$

[P] for every $n \geq 1$ since $\sup _{k}\left|X_{k}\right|<\infty$ and $\sum\left|a_{n k}\right|$ is convergent. Let $t>0$. We show $\mathrm{k} \geq \mathrm{l} \quad \mathrm{k} \geq \mathrm{l}$

that $\lim P\left\{\left|\sum_{n \rightarrow \infty} a_{n k}\left(X_{k}-E X_{k}\right)\right|>t\right\}=0$. Let $\varepsilon>0$. Since $X_{k}, k \geq 1$ is uniformly in$n \rightarrow \infty \quad k \geq 1$

tegrable, there exists $\delta>0$ such that

$$
\sup _{k \geq 1} \int_{A}\left|X_{k}\right| d P<\varepsilon t / 8 C
$$

whenever $A \in B$ and $P(A)<\delta$. Further, by Chebychev's inequality, for any $m>0$ and $\mathrm{k} \geq 1$,

$$
P\left\{\left|x_{k}\right|>m\right\} \leq(1 / m) E\left|x_{k}\right| \leq(1 / m) \sup _{k \geq 1} E\left|x_{k}\right| .
$$

Consequently, there exists a $>0$ such that

$$
\sup _{k \geq 1} P\left\{\left|X_{k}\right|>a\right\}<\delta \text {. }
$$

Define for every $k \geq 1$,

$$
\begin{aligned}
Y_{k} & =X_{k} \quad \text { if }\left|X_{k}\right| \leq a, \\
& =0 \quad \text { otherwise, and } \\
Z_{k} & =X_{k}-Y_{k} .
\end{aligned}
$$

Note that $Y_{k}, k \geq 1$ is a sequence of pairwise independent random variables satisfying $\left|Y_{k}-E Y_{k}\right| \leq 2 a$ for every $k \geq 1$. By $(2.1)$ and $(2.2)$, we have for every $k \geq 1$,

$$
E\left|z_{k}\right|=\int_{\left\{\left|x_{k}\right| \geq a\right\}}\left|x_{k}\right| d P<\varepsilon t / 8 C .
$$


Consequently, for every $\mathrm{n} \geq 1$,

$$
E\left|\sum_{k \geq 1} a_{n k}\left(Z_{k}-E Z_{k}\right)\right| \leq \sum_{k \geq 1}\left|a_{n k}\right| E\left|z_{k}-E Z_{k}\right| \leq 2 \sum_{k \geq 1}\left|a_{n k}\right| E\left|z_{k}\right|<\varepsilon t / 4 .
$$

Therefore, by Chebychev's inequality, for every $\mathrm{n} \geq 1$,

$$
P\left\{\left|\sum_{k \geq 1} a_{n k}\left(Z_{k}-E Z_{k}\right)\right|>t / 2\right\}<\varepsilon / 2 .
$$

Next, we choose $N \geq 1$ such that for every $n \geq N$, we have

$$
\max _{\mathrm{k} \geq 1}\left|a_{n k}\right|<\varepsilon \mathrm{t}^{2} / 32 \mathrm{a}^{2} \mathrm{C} .
$$

We observe that for every $n \geq N$,

$$
\begin{aligned}
& P\left\{\left|\sum_{k>1} a_{n k}\left(Y_{k}-E Y_{k}\right)\right|>t / 2\right\} \leq\left(4 / t^{2}\right) \operatorname{Var}\left(\sum_{k \geq 1} a_{n k}\left(Y_{k}-E Y_{k}\right)\right) \\
& =\left(4 / t^{2}\right) \underset{k \geq 1}{\sum_{n k}} a_{n}^{2} E\left(Y_{k}-E Y_{k}\right)^{2}
\end{aligned}
$$

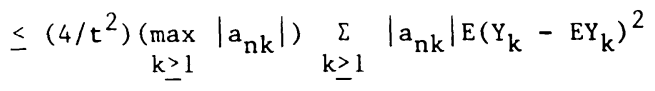

$$
\begin{aligned}
& <\varepsilon / 2
\end{aligned}
$$

Finally, (2.3) and (2.4) yield

$$
\begin{aligned}
P\left\{\left|\sum_{k \geq 1} a_{n k}\left(X_{k}-E X_{k}\right)\right|>t\right\} \leq & P\left\{\left|\sum_{k \geq 1} a_{n k}\left(Y_{k}-E Y_{k}\right)\right|>t / 2\right\} \\
& +P\left\{\left|\sum_{k \geq 1} a_{n k}\left(Z_{k}-E Z_{k}\right)\right|>t / 2\right\} \\
& <\varepsilon \text { for every } n \geq N .
\end{aligned}
$$

Thus $\sum a_{n k}\left(X_{k}-E X_{k}\right), n \geq 1$ converges to 0 in probability. $\mathrm{k} \geq 1$

To establish mean convergence, it suffices to show that $\underset{k>1}{\sum} a_{n k}\left(X_{k}-E X_{k}\right), n \geq 1$ is uniformly integrable. See Chung [2, Theorem 4.5.4, p.97] . Since $x_{k}, k \geq 1$ is uniformly integrable and $\sum_{k \geq 1}\left|a_{n k}\right| \leq C$ for every $n \geq 1$, it is obvious that $\underset{k \geq 1}{\sum} a_{n k}\left(X_{k}-E_{k}\right), n \geq 1$ is uniformly integrable.

REMARKS. (1). Rohatgi [7, Theorem 1, p. 305] showed that $\underset{k \geq 1}{\sum} a_{n k}\left(X_{k}-E x_{k}\right), n \geq 1$ converges to 0 in probability under the following conditions.

(i) $x_{n}, n \geq 1$ is independent.

(ii) (B) holds with $s=1$.

(iii) The double array $a_{n k}, n \geq 1, k \geq 1$ of real numbers satisfies (i) and (ii) of Theorem 1 .

In view of the remarks made in the introduction, Theorem 1 generalizes this result of Rohatgi. (Also, this result of Rohatgi was a generalization of a result of Pruitt [6, Theorem 1, p. 770] who started with the assumption that the sequence $x_{n}, n \geq 1$ is independently identically distributed with $\left.E\left|X_{1}\right|<\infty\right)$. Moreover, our proof is simpler than the one presented by Rohatgi. The essential difference in the proofs lies in the fact that we truncate each $x_{n}$ at a fixed point a, where as Rohatgi truncated $x_{n}$ at $a_{n}$ with $a_{n}$ varying with $n$. To illustrate the power of Theorem 1 over Theorem 1 of Rohatgi, consider the following example. Let $x_{n}, n \geq 1$ be a sequence of pairwise independent. random variable with $x_{n}$ having the following probability law. 


$$
\begin{aligned}
& P\left\{X_{n}=n\right\}=P\left\{X_{n}=-n\right\}=1 / 2 n \log (n+1), \\
& P\left\{X_{n}=0\right\}=1-(1 / n \log (n+1)) .
\end{aligned}
$$

$x_{n}, n \geq 1$ is uniformly integrable. But (B) does not hold for the sequence $x_{n}, n \geq 1$ with $s=1$. Rohatgi's theorem is not applicable to determine the convergence of $(1 / n) \sum_{k=1}^{n} x_{k}, n \geq 1$ to 0 in probability. But by Theorem 1 , the sequence $(1 / n) \sum_{k=1}^{n} x_{k}$, $\mathrm{n} \geq 1$ does indeed converge to 0 in probability.

(2). Chung [2, Theorem 5.2.2, p. 109] proved (attributed to Khintchine) the result that $(1 / n)\left(X_{1}+x_{2}+\ldots+x_{n}\right), n \geq 1$ converges to $E x_{1}$ in probability if $x_{n}, n \geq 1$ is a sequence of pairwise independent identically distributed random variables with $E\left|X_{1}\right|<\infty$. Theorem 1 generalizes this result, Moreover, the proof presented here is much simpler than the one presented by Chung.

If we impose a stronger condition on the double array, we can establish a Weak Law of Large Numbers for weighted sums without the assumption of independence of the random variables but in the presence of uniform integrability.

THEOREM 2. Let $X_{n}, n \geq 1$ be a sequence of real random variables defined on a probability space $(\Omega, \beta, P)$ such that $\left|x_{n}\right|^{r}, n \geq 1$ is uniformly integrable for some $0<r<1$. Let $a_{n k}, n \geq 1, k \geq 1$ be a double array of real numbers satisfying

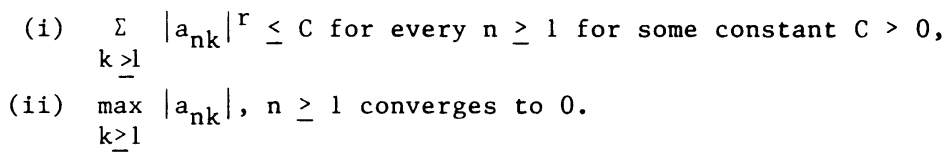

Then $\sum_{k \geq 1} a_{n k} X_{k}, n \geq 1$ converges to 0 in $r-t h$ mean.

PROOF. This can be proved by a simple modification of the proof of Theorem 1 . The series $\sum a_{n k} X_{k}, n \geq 1$ converges absolutely a.e. [P] for every $n \geq 1$ since $k \geq 1$

$\sum\left|a_{n k}\right|^{r}\left|X_{k}\right| r$ converges a.e. [P] and $0<r<1$. The inequality (2.1) takes the form $k \geq 1$

$$
\sup _{k \geq 1} \int_{A}\left|X_{k}\right|^{r} d P<\varepsilon t / 8 C
$$

and the inequality (2.2) remains intact as it is. The sequences $Y_{k}, k \geq 1$ and $z_{k}, k \geq 1$ are defined in exactly the same way as it was done in the above proof. The probability $P\left\{\left|\sum_{k>1} a_{n k} Z_{k}\right|>t / 2\right\}$ is estimated by $\sum_{k>1}\left|a_{n k}\right|^{r} E\left|z_{k}\right|^{r}$. Now comes the point of departure. In the proof of Theorem 1 , we showed that $\sum a_{n k}\left(Y_{k}-E Y_{k}\right), k \geq 1$ converges to zero $k \geq 1$

in probability. Under the conditions of Theorem 2, we can do better than this. The sequence $\sum_{k \geq 1} a_{n k} Y_{k}, n \geq 1$ does indeed converge to 0 a.e. [P]. This follows from the following chain of inequalities. For every $\mathrm{n} \geq 1$,

$$
\left.\left|\sum_{k \geq 1} a_{n k} \dot{Y}_{k}\right| \leq a \sum_{k \geq 1}\left|a_{n k}\right| \leq a \underset{k \geq 1}{a(\max }\left|a_{n k}\right|\right)^{1-r} \sum_{k \geq 1}\left|a_{n k}\right|^{r} .
$$

It now follows that $\sum a_{n k} x_{k}, n \geq 1$ converges to 0 in probability. To establish con$\mathrm{k} \geq 1$ 
vergence in $r$-th mean, it suffices to show that $\mid \underset{k \geq 1}{\sum_{n k}} a_{k} x_{k}, n \geq 1$ is uniformly integrable. This is not hard to prove.

REMARKS. Rohatgi [7, Theorem 1, p.305] established a weaker conslusion than the one given above under stronger conditions that the sequence $x_{n}, n \geq 1$ is independently distributed and that (B) holds for the sequence $x_{n}, n \geq 1$ with $s=r$. The major improvement achieved by Theorem 2 over Rohatgi's theorem is dropping the assumption of independence.

Now, we enquire about the validity of Theorems 1 and 2 in the context of separable Banach spaces. Theorem 2 is valid for sequences of random variables taking values in separable Banach spaces. For the sake of clarity, we give the statement below.

THEOREM 3. Let $x_{n}, n \geq 1$ be a sequence of random elements taking values in a separable Banach space $B$ equipped with a norm $\|$.$\| such that \left\|x_{n}\right\| r$, $n \geq 1$ is uniformly integrable for some $0<r<1$. Let $a_{n k}, n \geq 1, k \geq 1$ be a double array of real numbers satisfying (i) and (ii) of Theorem 2. Then $\sum a_{n k} X_{k}, n \geq 1$ converges to 0 in $k \geq 1$

the $\mathrm{r}$-th mean, i.e., $\mathrm{E}\left\|\sum_{\mathrm{k}} \mathrm{a}_{\mathrm{nk}} \mathrm{X}_{\mathrm{k}}\right\|^{\mathrm{r}}, \mathrm{n} \geq 1$ converges to zero.

REMARKS. (1). The proof of Theorem 3 is analogous to the one given for Theorem

2. This result is a generalization of Theorem 2.1 of [1]. The major improvement achieved in the above result is in disposing of the assumption of independence in Theorem 2.1 of [1]. Moreover, the proof suggested above is much simpler than the one presented in [1] for Theorem 2.1.

(2). Theorem 1 is not valid for Banach space-valued random variables under conditions similar to those imposed in Theorem 1. (See the comments following Theorem 1.1 of [1]). For the validity of Theorem 3, almost sure convergence of $\sum a_{n k} Y_{k}, n \geq 1$ $\mathrm{k}>\mathrm{l}$

to 0 certainly helped. The proof given for Theorem 1 fails to work in Banach spaces because we are unable to establish convergence of $\sum_{k>1} a_{n k} Y_{k}, n \geq 1$ to 0 either in probability or a.e. [P]. However, if $X_{n}, n \geq 1$ is uniformly tight, i.e., given $\varepsilon>0$, there exists a compact subset $C$ of $B$ such that $P\left\{X_{n} \in C\right\}>1-\varepsilon$ for every $n \geq 1$, then Theorem 1 is valid under the conditions stipulated therein. For further details on this result, see Wang and Bhaskara Rao [10, Theorem 2.4].

3. ON STRONG CONVERGENCE.

Extensions of Kolmogorov's Strong Law of Large Numbers are generally sought so that they become more applicable under circumstances less stringent than those imposed by Kolmogorov's Strong Law of Large Numbers. Jamison, Orey and Pruitt [4, Theorem 3, p. 42] worked with independent identically distributed sequences of random variables but imposed conditions on the weights to establish Strong Law of Large Numbers. Etemadi [3, Theorem 1, p. 119] relaxed the assumption of independence in Kolmogorov's Strong Law of Large Numbers to pairwise independence and arrived at the same conclusion. The following result encompasses both these extensions.

THEOREM 4. Let $x_{n}, n \geq 1$ be a sequence of pairwise independent identically distributed random variables defined on a probability space $(\Omega, \beta, P)$ satisfying $E\left|X_{1}\right|<\infty$. Let $a_{n}, n \geq 1$ be a sequence of positive numbers satisfying 1 im $\max \left(a_{i} / A_{n}\right)=0$, where $n \rightarrow \infty \quad 1 \leq i \leq n$ 
$A_{n}=\sum_{i=1}^{n} a_{i}, n \geq 1$. Let $N(n)$ be the number of positive integers $k \geq 1$ such that $\left(A_{k} / a_{k}\right) \leq n, n \geq 0$. If $N(n) / n \leq r$ for every $n \geq 1$ for some constant $r>0$, then $\sum_{k=1}^{n}\left(a_{k} / A_{n}\right) X_{k}, n \geq 1$ converges to $\operatorname{Ex}_{1}$ a.e. $[P]$.

PROOF. The proof is carried out in the following three major steps. $1^{\circ}$. Since each of the sequences $x_{n}^{+}, n \geq 1$ and $x_{n}^{-}, n \geq 1$ satisfies the hypothesis of the theorem, we can assume, without loss of generality, that each $X_{n} \geq 0$. For each $k \geq 1$, let

$$
\begin{aligned}
\mathrm{Y}_{\mathrm{k}} & =\mathrm{X}_{\mathrm{k}} & & \text { if } \mathrm{X}_{\mathrm{k}}<\mathrm{A}_{\mathrm{k}} / \mathrm{a}_{\mathrm{k}}, \\
& =0 & & \text { otherwise. }
\end{aligned}
$$

Note that $Y_{k}, k \geq 1$ is a sequence of pairwise independent random variables. It can be shown that $\sum_{n \geq 1}\left(a_{n}^{2} / A_{n}^{2}\right) E Y_{n}^{2}$ is convergent. Further, $\sum_{k=1}^{n}\left(a_{k} / A_{n}\right) X_{k}, n \geq 1$ converges to $E_{1}$ a.e.[P] if and only if $\sum_{k=1}^{n}\left(a_{k} / A_{n}\right)\left(Y_{k}-E Y_{k}\right), n \geq 1$ converges to 0 a.e. [P]. The details are worked out in Stout [8, p. 221].

$2^{\circ}$. We now show that almost sure convergence takes place along some we 11 chosen special subsequences of $\sum_{k=1}^{n}\left(a_{k} / A_{n}\right)\left(Y_{k}-E Y_{k}\right), n \geq 1$. In the final step $3^{0}$, we will show that convergence takes place along the entire sequence almost surely. Let $\alpha>1$. Define a sequence $\mathrm{m}_{1}, \mathrm{~m}_{2}, \ldots$ of positive integers by letting $\mathrm{m}_{1}=1$ and $\mathrm{m}_{i}=\mathrm{min}$ $\left\{j \geq 1 ; \quad A_{j} \geq A_{m_{i-1}} \alpha\right\}, i=2,3, \ldots$. Obviously, $m_{1}<m_{2}<m_{3}<\ldots$. We show that $\sum_{k=1}^{m_{i}}\left(a_{k} / A_{m_{i}}\right)\left(Y_{k}-E Y_{k}\right), i \geq 1$ converges to 0 a.e.[P]. It suffices to show that for any $\varepsilon>0$,

$$
\mathrm{B}_{\alpha, \varepsilon}=\sum_{i \geq 1} P\left\{\left|\sum_{k=1}^{m_{i}}\left(a_{k} / A_{m_{i}}\right)\left(Y_{k}-E Y_{k}\right)\right| \geq \varepsilon\right\}<\infty .
$$

By Chebychev's inequality,

$$
\begin{aligned}
& B_{\alpha, \varepsilon} \leq\left(1 / \varepsilon^{2}\right) \sum_{i \geq 1}^{\sum_{k=1}} \operatorname{Var}\left(\sum_{k=1}^{m_{i}}\left(a_{k} / A_{m_{1}}\right)\left(Y_{k}-E Y_{k}\right)\right) \\
& =\left(1 / \varepsilon^{2}\right) \sum_{i \geq 1}^{\sum_{i}} \sum_{k=1}^{m_{k}}\left(a_{k}^{2} / A_{m_{i}^{2}}^{2} \operatorname{Var}\left(Y_{k}\right)\right. \\
& \leq\left(1 / \varepsilon^{2}\right) \sum_{i \geq 1} \sum_{k=1}^{m_{i}}\left(a_{k}^{2} / A_{m_{i}^{2}}^{2} E Y_{k}^{2}\right. \\
& =\left(1 / \varepsilon^{2}\right) \sum_{k \geq 1}^{\sum} a_{k}^{2} E Y_{k}^{2} \sum_{j \geq j} 1 / A_{m_{j}}^{2}, \\
& \text { where } j_{k}=\min \left\{j \geq 1 ; m_{j} \geq k\right\}, j=1,2,3, \ldots .
\end{aligned}
$$

It is clear that $A_{m_{j}} \geq A_{k}$ and $\sum_{j \geq j_{k}} 1 / A_{m_{j}}^{2} \leq\left(1 / A_{m_{j}}^{2}\right)\left(1+1 / \alpha^{2}+1 / \alpha^{4}+1 / \alpha^{6}+\ldots\right)$

$=\left(1 / \mathrm{A}_{\mathrm{m}_{\mathrm{k}}}^{2}\right)\left(\alpha^{2} / \alpha^{2}-1\right) \leq\left(1 / \mathrm{A}_{\mathrm{k}}^{2}\right)\left(\alpha^{2} / \alpha^{2}-1\right)$.

Consequently,

$$
\mathrm{B}_{\alpha, \varepsilon} \leq\left(1 / \varepsilon^{2}\right)\left(\alpha^{2} / \alpha^{2}-1\right) \sum_{k \geq 1}\left(a_{k}^{2} / A_{n}^{2}\right) \mathrm{EY}_{k}^{2}<\infty \text {, by } 1^{\circ} .
$$

$3^{0}$. Finally we show that the entire sequence $\sum_{k=1}^{n}\left(a_{k} / A_{n}\right)\left(Y_{k}-E Y_{k}\right) n \geq 1$ converges to 0 a.e.[P]. Since $\lim _{n \rightarrow \infty} \max _{1 \leq k \leq n}\left(a_{k} / A_{n}\right)=0, \lim _{n \rightarrow \infty}\left(a_{n} / A_{n-1}\right)=0$ and $\lim _{n \rightarrow \infty}\left(A_{n} / A_{n-1}\right)=1$. 
Observe also that $A_{n} / A_{n-1}>1$ for every $n \geq 2$. We can find an integer $N_{\alpha} \geq 1$ such that $1<\left(A_{n} / A_{n-1}\right)<\alpha$ for all $n \geq N_{\alpha}$. Equivalently, if $n \geq N_{\alpha}, A_{n-1}<A_{n}<\alpha A_{n-1}$. For each $\mathrm{n} \geq 1$, there exists a unique integer $\mathrm{i} \geq 2$ such that $\mathrm{m}_{i-1} \leq \mathrm{n}<\mathrm{m}_{i}$. If $\mathrm{m}_{i-1} \geq \mathrm{N}_{\alpha}$, then

$$
A_{n}<A_{m_{i}}<\alpha A_{m_{i}-1}<\alpha^{2} A_{m_{i-1}}<\alpha^{2} A_{n} \text {. }
$$

Consequently, $1 / A_{n}<\alpha^{2} / A_{m_{i}}$ and $\underset{k=1}{n}\left(a_{k} / A_{n}\right) Y_{k} \leq \alpha^{2} \sum_{k=1}^{m_{i}}\left(a_{k} / A_{m_{i}}\right) Y_{k}$ for every $n \geq N_{\alpha}$.

Therefore,

$$
\underset{n \rightarrow \infty}{\lim \sup _{k=1}} \sum_{k=1}^{n}\left(a_{k} / A_{n}\right) Y_{k} \leq \lim _{i \rightarrow \infty} \sum_{k=1}^{m_{i}}\left(a_{k} / A_{m_{i}}\right) Y_{k}=\alpha^{2} E x_{1} \text { a.e. [P]. }
$$

(One can check that $\lim _{n \rightarrow \infty} E_{n}=E X_{1}$ and, by Toeplitz lemma, $\lim _{n \rightarrow \infty} \sum_{k=1}^{n}\left(a_{k} / A_{n}\right) E Y_{k}=E X_{1}$.) From (3.1), we also observe that $1 / A_{n}>1 / \alpha^{2} A_{m_{i-1}}$ and $\sum_{k=1}^{n}\left(a_{k} / A_{n}\right) Y_{k} \geq\left(1 / \alpha^{2}\right) \sum_{k=1}^{m_{1-1}}$ $\left(a_{k} / A_{m_{i-1}}\right) Y_{k}$, if $m_{i-1} \geq N_{\alpha}$. Consequently, $\underset{n \rightarrow \infty}{\lim \inf } \sum_{k=1}^{n}\left(a_{k} / A_{n}\right) Y_{k} \geq(1 / \alpha 2) \lim _{i \rightarrow \infty} \sum_{k=1}^{m_{i}-1}\left(a_{k} / A_{m_{i-1}}\right) Y_{k}=\left(1 / \alpha^{2}\right) E_{1}$ a.e. [P]. Thus we observe that for every $\alpha>1$, $\left(1 / \alpha^{2}\right) \mathrm{EX}_{1} \leq 1 \mathrm{im} \inf _{\mathrm{n} \rightarrow \infty} \sum_{k=1}^{n}\left(a_{k} / A_{n}\right) Y_{k} \leq 1$ im $\sup _{n \rightarrow \infty} \sum_{k=1}^{n}\left(a_{k} / A_{n}\right) Y_{k} \leq \alpha^{2} E X_{1}$ a.e. [P]. This proves the almost sure convergence of the desired sequence.

REMARKS. (1). This theorem extends to separable Banach spaces-valued random variables verbatim. A proof can easily be obtained with appropriate modifications of the proof of Strong Law of Large Numbers given in Padgett and Taylor [5, p.42-44]. 0r, one can adopt the argument given in Bozorgnia and Bhaskara Rao [1] in the proof of their Theorem 2.1.

(2). Kolmogorov's inequality plays a crucial role in the standard proof offered in many teyt books for Kolmogorov's Strong Law of Large Numbers. This inequality, as it stands, cannot be commandeered for pairwise independent sequences of random variables. The idea of establishing convergence along some special subsequences is taken from Etemadi [3] but his technique has been modified extensively in the above proof to suit our needs. Also, Theorem 4 strengthens the conclusion of Theorem 5.2.2 of Chung [2, p. 109] from convergence in probability to convergence almost everywhere [P].

There are sequences $a_{n}, n \geq 1$ of positive numbers such that $\lim _{n \rightarrow \infty} \max a_{k} / A_{n}=0$ but $N(n) / n, n \geq 1$ is unbounded. See Jamison, Orey and Pruitt [4, p.43]. In such a case, Theorem 4 becomes inapplicable. However, if we impose a stronger condition that $\mathrm{E}\left|\mathrm{X}_{1}\right| \log ^{+}\left|\mathrm{X}_{1}\right|<\infty$, one can establish a Strong Law of Large Numbers generalizing Theorem 4 of Jamison, Orey and Pruitt [4, p.43] as follows.

THEOREM 5. Let $x_{n}, n \geq 1$ be a sequence of pairwise independent identically distributed real random variables defined on a probability space $(\Omega, \beta, P)$ with $E\left|x_{1}\right| \log ^{+}\left|x_{1}\right|<\infty$. Let $a_{n}, n \geq 1$ be a sequence of positive numbers satisfying $\underset{n \rightarrow \infty}{\lim } A_{n}=\infty$, where $A_{n}=\sum_{i=1}^{n} a_{i}, n \geq 1$. Then $\sum_{k=1}^{n}\left(a_{k} / A_{n}\right) X_{k}, n \geq 1$ converges to $\operatorname{EX}_{1}$ a.e. $[P]$. 
PROOF. Using Lemma 2 of Jamison, Orey and Pruitt [4, p.43], one can prove the above result by a suitable modification of the proof of Theorem 4 .

REMARK. Theorem 5 is also valid for separable Banach space-valued random variables. The relevant moment condition is that $E|| X_{1}|| \log ^{+}|| X_{1}||<\infty$.

ACKNOWLEDGEMENTS. The authors are grateful to the referee for his comments and his suggestions to improve the tone of the paper.

Part of the work of the second author was supported by the Air Force office of Scientific Research under Contract F49620-82-0001. Reproduction in whole or in part is permitted for any purpose of the United States Government.

\section{REFERENCE}

1. BOZORGNIA, A. and BHASKARA RAO, M. Limit theorems for Weighted Sums of Random Elements in Separable Banach Spaces, J. Multivariate Anal., $\underline{9}$ (1979), 428-433.

2. CHUNG, K.L. A Course in Probability Theory, Second Edition, Academic Press, New York, 1974.

3. ETEMADI, N. An Elementary Proof of the Strong Law of Large Numbers, Z. Wahrscheinlichkeitstheorie Verw. Gebiete, 55 (1981), 119-122.

4. JAMISON, B., OREY, S. and PRUITT, W. Convergence of Weighted Averages of Independent Random Variables, Z. Wahrscheinlichkeitstheorie Verw. Gebiete, 4 (1965), 40-44.

5. PADGETT, W. and TAYLOR, R.L. Laws of Large Numbers for Normed Linear Spaces and certain Frechet Spaces, Lecture Notes in Mathematics No. 360, Springer-Verlag, Berlin, 1973 .

6. PRUITT, W.E. Summability of Independent Random variables, J. Math. Mech., 15 (1966), 769-776.

7. ROHATGI, V.K. Convergence of weighted sums of independent random variables, Proc. Camb. Phil. Soc., 69 (1971), 305-307.

8. STOUT, W.F. Almost sure convergence, Academic Press, New York, 1974.

9. WEI, W. and TAYLOR, R.L. Convergence of Weighted Sums of Tight Random Elements, J. Multivariate Ana1., $\underline{8}(1978), 282-294$.

10. WANG, X.C. and BHASKARA RAO, M. Convergence in the p-th mean and Some Weak Laws of Large Numbers for Weighted Sums of Random Elements in Separable Normed Linear Spaces, to J. Multivariate Ana1., 15 (1984), 124-134. 


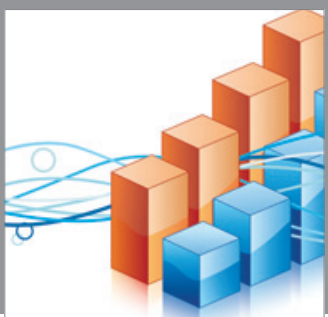

Advances in

Operations Research

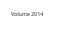

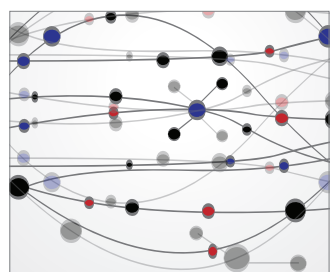

\section{The Scientific} World Journal
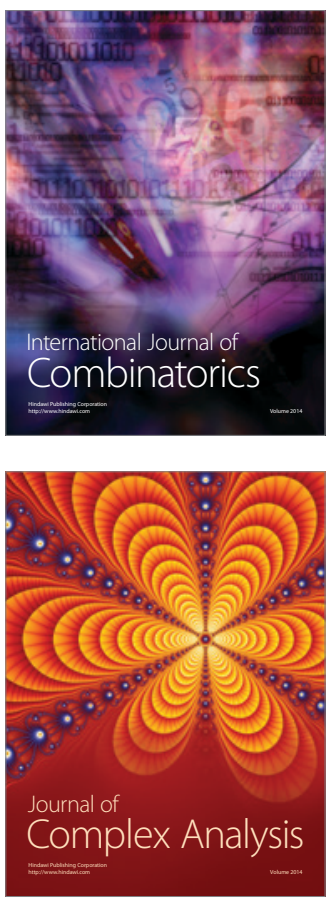

International Journal of

Mathematics and

Mathematical

Sciences
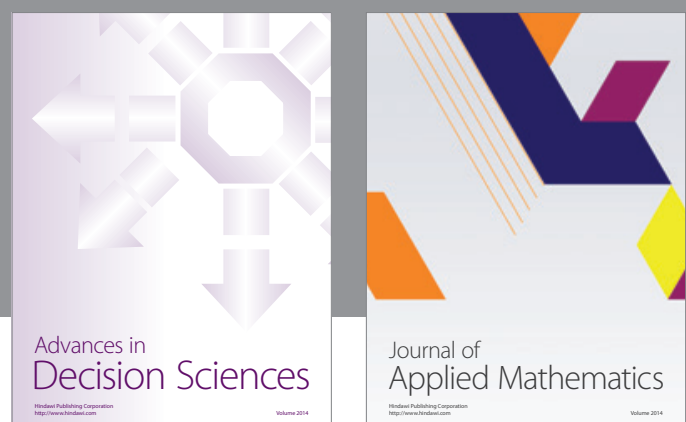

Journal of

Applied Mathematics
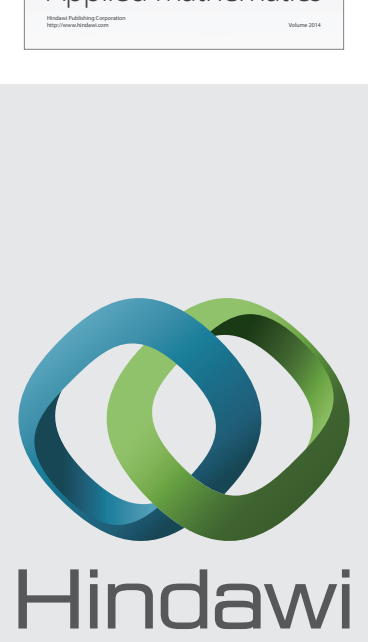

Submit your manuscripts at http://www.hindawi.com
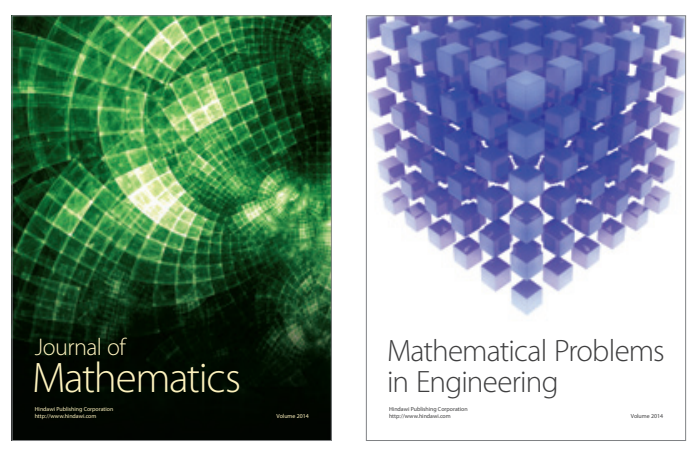

Mathematical Problems in Engineering
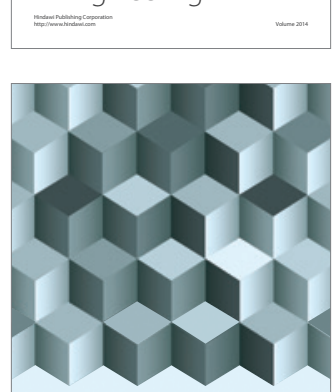

Journal of

Function Spaces
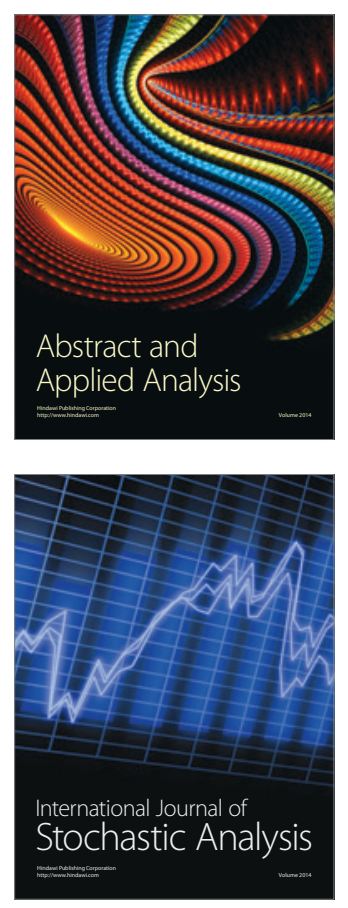

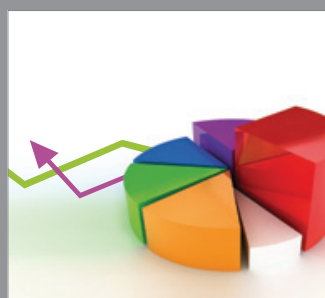

ournal of

Probability and Statistics

Promensencen
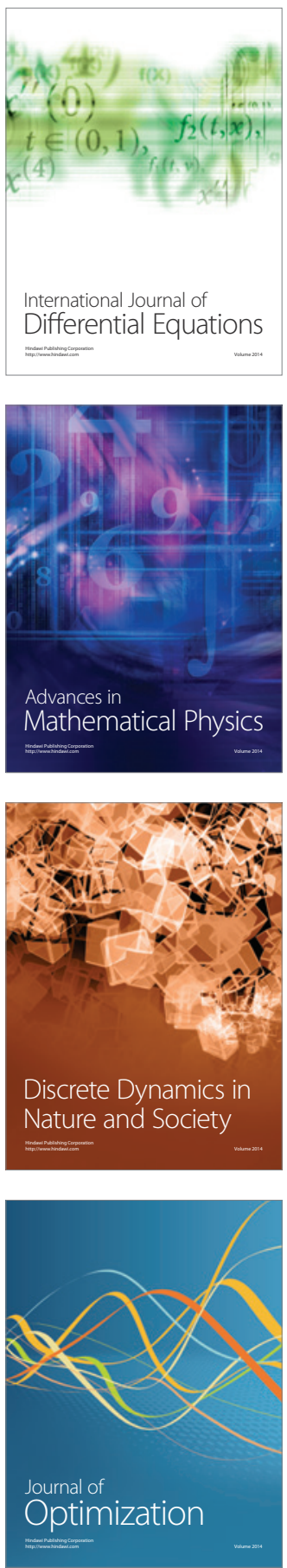